Artículo Original. Influencia del entrenamiento interválico de alta intensidad en la capacidad aeróbica de personas con lesión medular. Vol. $5, n^{\circ}$. 2; p. 190-212, mayo 2019. A Coruña. España ISSN 2386-8333
\end{abstract}

\title{
Influencia del entrenamiento interválico de alta intensidad en la capacidad aeróbica de personas con lesión medular
}

\author{
Influence of high-intensity interval training on the aerobic capacity of persons with \\ spinal cord injury
}

\author{
Joan Rodríguez Barnada; Sara Alonso Guntín; Lidia Guerrero Sánchez; Xavier Redón \\ Castellví.
}

Centros Puente Fundació Isidre Esteve

centroponte@fundacioisidreesteve.org

\begin{abstract}
Cronograma editorial: Artículo recibido: 28/08/2018 Aceptado: 21/04/2019 Publicado: 01/05/2019
DOI: https://doi.org/10.17979/sportis.2019.5.2.3919
\end{abstract}

\section{Resumen}

En las personas con lesión medular se reduce la capacidad funcional y de llevar a cabo las tareas de la vida diaria. La principal causa de muerte en la fase crónica son las enfermedades cardiovasculares.La capacidad aeróbica es una medida indirecta del riesgo de enfermedad cardiovascular. El entrenamiento de resistencia mejora esta capacidad.

El objetivo del estudio fue analizar el efecto de un entrenamiento interválico de alta intensidad (HIIT) en 7 participantes con lesión medular (LM), Asia A (escala de afectación neurológica) y en fase crónica (más de 6 meses tras la lesión). Sabiendo que el entrenamiento continuo mejora la capacidad aeróbica, comprobaremos si el interválico también lo hace.

La intervención fue precedida de un test donde se registró: peso, perímetro de cintura, frecuencia cardíaca en reposo (FCR) y cuestionario DASH. De manera activa en un ergómetro modelo Skierg Concept 2 (Vermont, 2009) se registró potencia máxima absoluta, potencia máxima relativa, y potencia media absoluta y relativa, en 1 minuto y 6 minutos.

El programa se realizó durante cuatro semanas, a razón de tres sesiones por semana (lunes, miércoles y viernes). Al finalizar las doce sesiones se realizó un post-test donde se observó la mejora en potencia máxima absoluta y relativa, así como en las pruebas de 1 y 6 minutos. Los datos fueron analizados con SPSS utilizando una t-student para muestras relacionadas con una significación del 95\% ( $p<0.05$ ). Los resultados nos invitan a seguir investigando sobre este tipo de entrenamiento en personas con lesión medular ya que pueden

Para citar este artículo utilice la siguiente referencia: Rodríguez-Barcala, j.; Guerrero-Sánchez, 1.; Redón, X.. (2019). Influencia del entrenamiento

interválico de alta intensidad en la capacidad aeróbica de personas con lesión medular. Sportis Sci J, 5 (2), 190-212.

DOI: https://doi.org/10.17979/sportis.2019.5.2.3919

http://revistas.udc.es/ 
Artículo Original. Influencia del entrenamiento interválico de alta intensidad en la capacidad aeróbica de personas con lesión medular. Vol. $5, n^{\circ}$. 2; p. 190-212, mayo 2019. A Coruña. España ISSN 2386-8333

influir positivamente en su capacidad aeróbica, repercutiendo en su calidad de vida y disminuyendo el riesgo de enfermedad cardiovascular.
\end{abstract}

\title{
Palabras clave
}

Entrenamiento, HIIT, lesión medular, resistencia, aeróbico.

\begin{abstract}
People with spinal cord injuries have reduced functional capacity and difficulties on daily life tasks. The greatest cause of death on chronic cases is cardiovascular disease.

The aerobic capacity is a non direct measure of having cardiovascular diseases risk. Resistance training could highly improve that capacity. The aim of the present study was to analyse the effect of a high intensity interval training (HIIT) in a sample of 7 participants with spinal cord injury (SCI), Asia A (scale of neurological involvement) and chronical phase (more than 6 months after the injury). Knowing that training regularly will improve the aerobic capacity, we are going to check if intervalic training also works.

Before starting, we recorded their weight, waist circumference, resting heart rate (HRR) and Dash pain scale. While active we recorded in a Skierg Concept 2 (Vermont, 2009) model ergometer absolute maximum and relative power as well as absolute average power and relative average power in 1 minute and 6 minutes. The program went on for four weeks, training 3 times a week (Monday, Wednesday and Friday). Once the 12 sessions were over, a post-test was done. Here we observed that all participants improved their absolute and relative power, as well as in the 1 and 6 minutes tests. The data was analysed with SPSS using a paired t-test with a significance of $95 \%$ ( $p<0.05$ ).

These results encourages us to continue investigating about this type of training with people with spinal cord injuries since they can positively influence their aerobic capacity, prevent cardiosvascular diseases as well as having a positive impact on their quality of life.
\end{abstract}

\section{Key words}

Training, HIIT, Spinal cord injury, Endurance, Aerobic.

\section{Introducción}

En el ámbito de la discapacidad física, la lesión medular (LM) es una de las situaciones clínicas más devastadoras, tanto por pérdida funcional como de independencia.

La principal causa de muerte en la fase crónica son las enfermedades cardiovasculares (Giner, 2006; Myers, Lee y Kiratli, 2007). La capacidad aeróbica es una medida indirecta del riesgo de enfermedad cardiovascular (Kohl, 2001) y se ha comprobado una asociación entre el nivel de la lesión y el VO2max. (Van den Berg-Emons, 2008).

Para citar este artículo utilice la siguiente referencia: Rodríguez-Barcala, j.; Guerrero-Sánchez, 1.; Redón, X.. (2019). Influencia del entrenamiento interválico de alta intensidad en la capacidad aeróbica de personas con lesión medular. Sportis Sci J, 5 (2), 190-212.

DOI: https://doi.org/10.17979/sportis.2019.5.2.3919

http://revistas.udc.es/ 


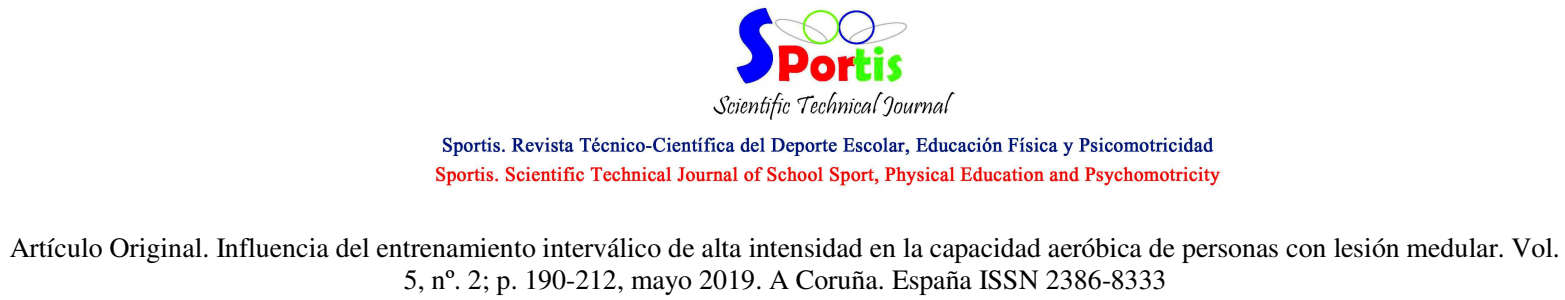

La baja capacidad aeróbica está asociada con la incapacidad para realizar actividades de la vida diaria, mayor frecuencia de infecciones de orina y menor satisfacción con la vida. (Hjeltnes y Jansen, 1990; van Koppenhagen et al., 2014).

El pedaleo de brazos es un ejercicio aeróbico efectivo para personas que no pueden utilizar los miembros inferiores (Hicks, 2003) y se ha demostrado que su práctica regular tiene efectos positivos sobre el sistema cardiorrespiratorio (Valent et al., 2009; Brizuela, Sinz, Aranda y Martínez, 2010; Hicks et al., 2010), sobre la resistencia muscular y, además aumenta la capacidad física y la fuerza muscular (Hicks et al.,2010; Hicks et al., 2003; Brizuela, Sinz, Aranda y Martínez, 2010).

El $67 \%$ de la población con LM refiere dolor desde que va en silla, el $91 \%$ ha padecido dolor durante más de 3 meses seguidos y el 74\% durante más de un año (Alm et al., 2008). Parece evidente que la participación deportiva no incrementa el riesgo de dolor o lesiones en el hombro (Finley y Rodgers, 2004) sino que, por el contrario, los usuarios de silla de ruedas más sedentarios tienen un mayor riesgo de padecerlos en comparación con los deportistas (Fullerton et al., 2003).

El objetivo de este estudio es demostrar la efectividad de un programa interválico de alta intensidad en LM orientado a la mejora de los parámetros relacionados con la capacidad cardiovascular. Para ello, se busca evidenciar la mejora de valores absolutos y relativos en relación a las vías aeróbica (test de 6 minutos) y anaeróbica (test de 1 minuto) tras doce sesiones de entrenamiento interválico de alta intensidad (HIIT).

\section{Material y método}

Se trata de un estudio cuasi-experimental de antes y después, longitudinal. Se realizaron dos mediciones una previa a la intervención y otra posterior, y en el estudio no se utilizó un grupo control. Para este trabajo se han seguido los principios éticos citados en la declaración de Helsinki para la experimentación científica con seres humanos.

\section{Participantes}

Siete personas $(\mathrm{N}=7)$ con lesión medular $(\mathrm{LM})$ llevaron a cabo un programa de entrenamiento interválico de alta intensidad durante cuatro semanas, entre los meses septiembre y diciembre de 2017. Participaron tres mujeres y cuatro hombres con edades

Para citar este artículo utilice la siguiente referencia: Rodríguez-Barcala, j.; Guerrero-Sánchez, 1.; Redón, X.. (2019). Influencia del entrenamiento interválico de alta intensidad en la capacidad aeróbica de personas con lesión medular. Sportis Sci J, 5 (2), 190-212.

DOI: https://doi.org/10.17979/sportis.2019.5.2.3919

http://revistas.udc.es/ 
Artículo Original. Influencia del entrenamiento interválico de alta intensidad en la capacidad aeróbica de personas con lesión medular. Vol. 5, nº. 2; p. 190-212, mayo 2019. A Coruña. España ISSN 2386-8333

comprendidas entre 26 y 59 años. Todos ellos llevaban al menos 2 años de práctica deportiva regular.

Los participantes del estudio tenían una lesión medular ASIA A. La altura de la lesión estaba comprendida entre L1-T12 (el participante con la lesión más baja) y C6-C7 (la más alta).

La antigüedad de la lesión oscilaba entre los 14 años del participante con la lesión más antigua y 2 años de la más reciente, en el momento de iniciar del entrenamiento.

Tabla1. Datos estadísticos del estudio

Estadísticos descriptivos

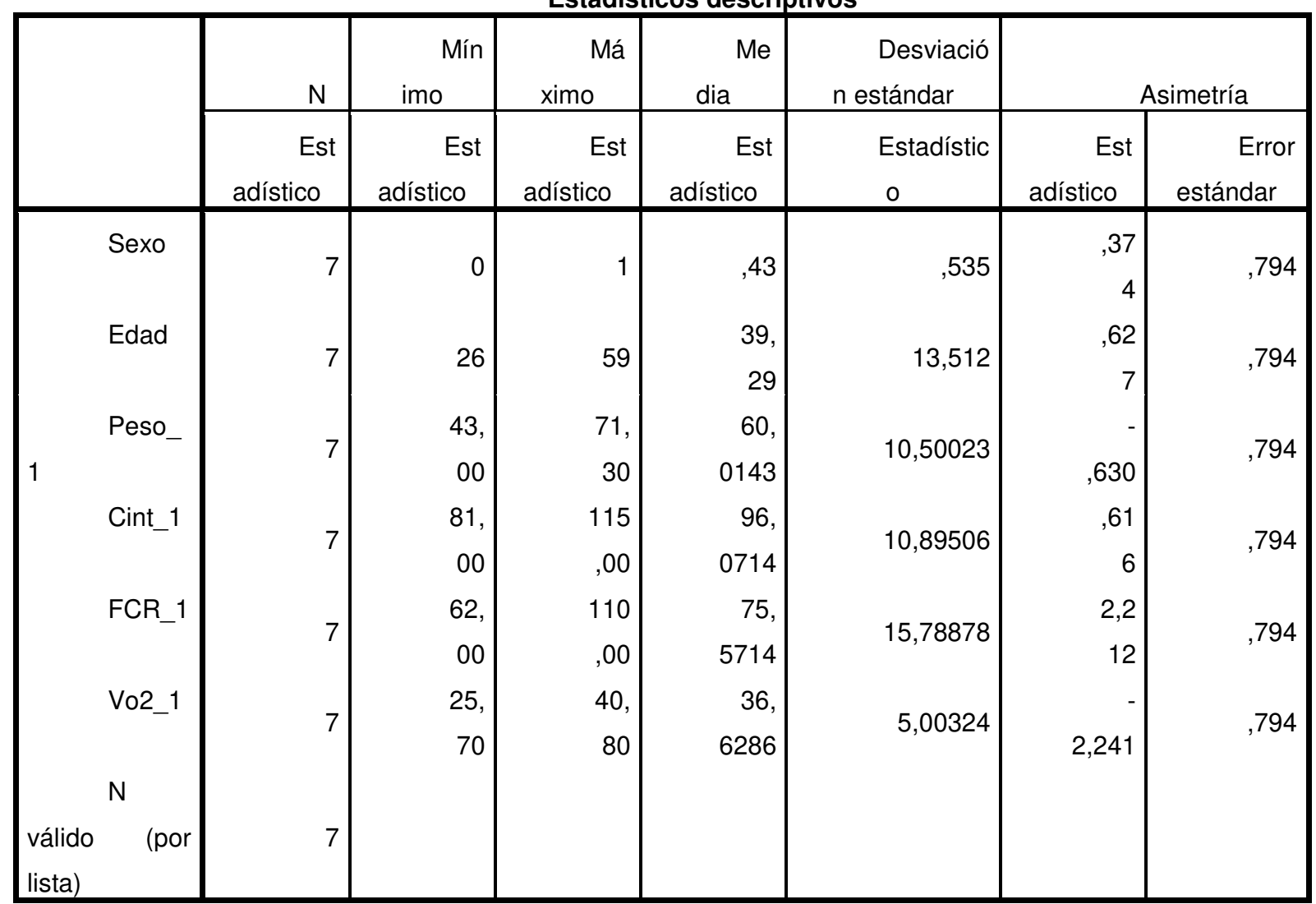

\section{Mediciones antropométricas y fisiológicas}

Antes de comenzar el programa se registró peso, perímetro de cintura y frecuencia cardíaca en reposo (FCR). Todos los participantes firmaron un documento de consentimiento informado previo a la realización del estudio donde se le explicaba todos los riesgos y 
Artículo Original. Influencia del entrenamiento interválico de alta intensidad en la capacidad aeróbica de personas con lesión medular. Vol. $5, n^{\circ}$. 2; p. 190-212, mayo 2019. A Coruña. España ISSN 2386-8333

beneficios de la investigación, en forma libre, sin presiones de ninguna índole y con el conocimiento que podían retirarse de la investigación cuando quisieran.

Para la medición del peso corporal se utilizó una báscula TANITA BF-350. Los participantes se sentaron sin apoyo de piernas ni de brazos para pesarse.

Para medir el perímetro de cintura se utilizó una cinta métrica $(\mathrm{mm})$. La medida se tomó pasando la cinta a la altura del ombligo y paralela al suelo.

Para registrar la FCR se colocó una banda torácica, monitorizada con un medidor de frecuencia cardíaca Polar M400. Para ello, se situó al participante en una sala con un ambiente tranquilo, sentado en su silla, durante 10 minutos controlando la frecuencia cada 5 segundos y tomando el resultado más bajo, después de registrar los 6 valores consecutivos con menor valor. (Fiona et al., 2011).

Para la estimación del VO2max. se utilizó la siguiente fórmula: (FC máx./FC Reposo) x 15 (Uth, Sørensen, Overgaard y Pedersen, 2004).

Para calcular la FC máxima se utilizó la ecuación: 208,75-0,73edad (Marins, Delgado y Benito, 2013).

\section{Test de aptitud cardiorrespiratoria}

Los test se efectuaron en el ergómetro Skierg Concept 2 PM5 (Vermont, 2009) sentados en su propia silla.

Potencia máxima: La prueba consiste realizar 15 paladas a la máxima intensidad posible. La resistencia del ergómetro la elige el propio participante. Se toma la mejor medición de 3 intentos con una recuperación de 2 minutos entre ellos. Se realizan 15 paladas ya que necesitan más tiempo de aceleración que cuando se realiza la prueba con personas sin LM. Para la potencia máxima absoluta se toma el valor más alto alcanzado y para la relativa se divide este resultado por el peso corporal.

Potencia media en 1 minuto: La prueba consiste en realizar 1 minuto a la máxima intensidad posible, tras haber descansado 5 minutos desde la finalización del primer test. La resistencia del ergómetro la elige el propio participante. Se realiza un solo intento para esta prueba y se registra la potencia media al final de la prueba. Para el registro de la potencia media absoluta se toma el valor medio del minuto, y para la potencia media relativa se divide ese valor entre el peso corporal del participante.

Para citar este artículo utilice la siguiente referencia: Rodríguez-Barcala, j.; Guerrero-Sánchez, 1.; Redón, X.. (2019). Influencia del entrenamiento interválico de alta intensidad en la capacidad aeróbica de personas con lesión medular. Sportis Sci J, 5 (2), 190-212. DOI: https://doi.org/10.17979/sportis.2019.5.2.3919

http://revistas.udc.es/ 
Artículo Original. Influencia del entrenamiento interválico de alta intensidad en la capacidad aeróbica de personas con lesión medular. Vol. $5, n^{\circ} .2 ;$ p. 190-212, mayo 2019. A Coruña. España ISSN 2386-8333

Potencia media en 6 minutos: La prueba consiste en realizar 6 minutos a la máxima intensidad posible, tras haber descansado 10 minutos desde la finalización del primer test. La resistencia del ergómetro la elige el propio participante. Se realiza un solo intento para esta prueba y se registra la potencia media al final de la prueba. Para el registro de la potencia media absoluta se toma el valor medio del minuto, y para la potencia media relativa se divide ese valor entre el peso corporal del participante.

Todos los test fueron monitorizados con un medidor de frecuencia cardíaca Polar M400. En todos los test y entrenamientos se preguntaba a los participantes por el nivel de esfuerzo, al finalizar, con la escala de valoración subjetiva del esfuerzo OMNI-GSE (Da Silva- Grigoletto, 2013).

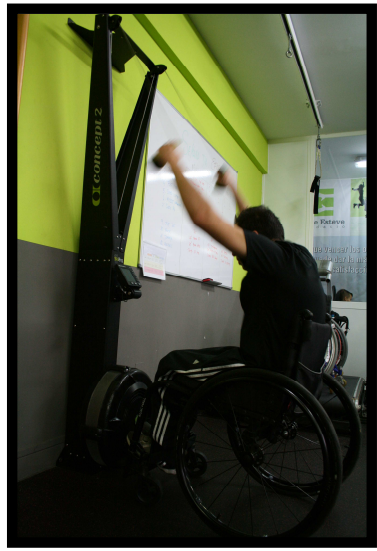

Figura 1. Skierg

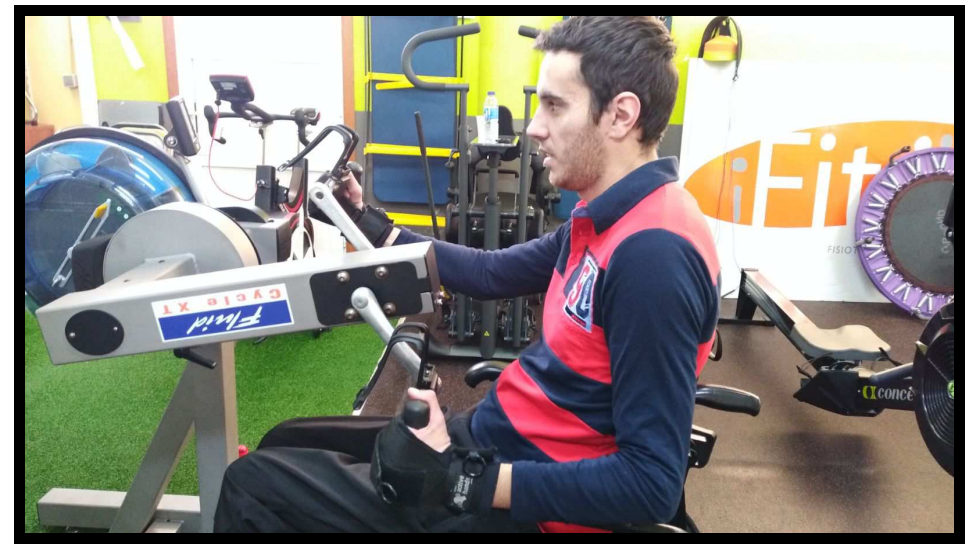

Figura 2. Bicicleta de manos

Las variables dependientes fueron: potencia máxima absoluta y relativa; potencia media absoluta y relativa, en 1 y 6 minutos; Fc reposo, Fc máxima; VO2max estimado y perímetro de cintura. Las variables independientes fueron las siguientes: fecha de nacimiento, fecha de inicio y sexo. Y como variables extrañas (no se registraron): Dieta y todo tipo de actividades realizadas durante el día.

Se utilizaron las pruebas de:

- 6 minutos para la vía aeróbica, ya que es la más validada en la bibliografía para deportistas que se desplazan en silla de ruedas y realizan la prueba en ergómetro de manos (Mcdonald, M. J., 2016), 
Artículo Original. Influencia del entrenamiento interválico de alta intensidad en la capacidad aeróbica de personas con lesión medular. Vol. $5, \mathrm{n}^{\circ}$. 2; p. 190-212, mayo 2019. A Coruña. España ISSN 2386-8333

- 1 minuto para la vía anaeróbica, debido a que los participantes con LM tardan bastante más tiempo en acelerar el ergómetro. Por ello consideramos que un Wingate es poco fiable y se convierte en una prueba demasiado corta para que alcancen una intensidad máxima en un tiempo breve.

\section{Programa de entrenamiento}

Los participantes hicieron un programa de entrenamiento interválico de resistencia de alta intensidad de 4 semanas. Realizaron tres sesiones semanales para un total de doce.

Calentamiento: En la primera parte del entrenamiento todos los participantes realizaban un calentamiento específico compuesto por: movilidad de cintura escapular, calentamiento cardiovascular, estabilización central y ejercicio compensatorio de hombro.

Tabla 2. Calentamiento del programa de entrenamiento

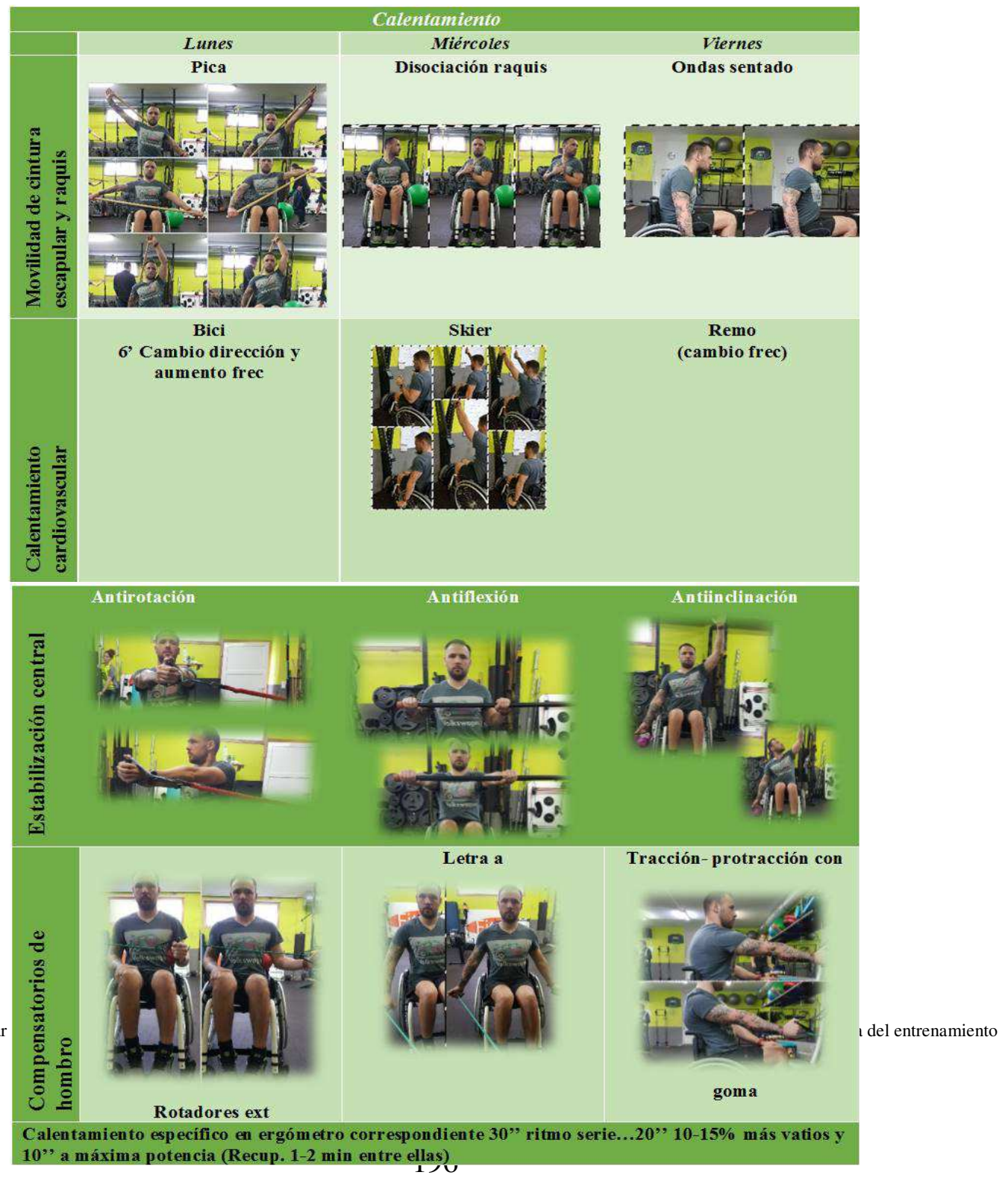




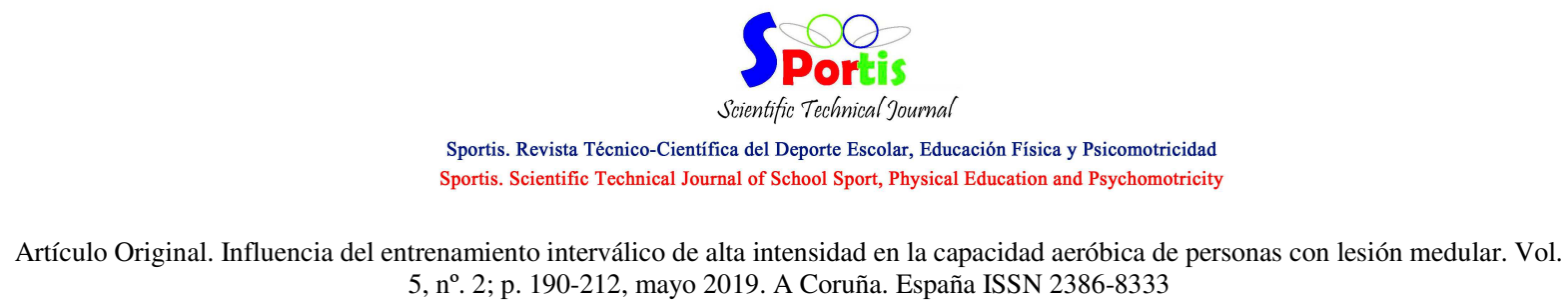
5, no. 2; p. 190-212, mayo 2019. A Coruña. España ISSN 2386-8333

Parte principal: El entrenamiento se realizaba lunes, miércoles y viernes y alternaba una sesión en skierg y otra de bici de manos.

El entrenamiento se dividió en 4 microciclos. El primer microciclo comenzaba con una sesión en Skierg de series de 1 minuto al $75 \%$ del test de 1 minuto, seguido de un entrenamiento en bici de series de 8 ' al $95 \%$ del test de 6 minutos. Las recuperaciones de estas series eran siempre pasivas. La tercera sesión de la semana corresponde a unos cambios de ritmo en Skierg, donde la intensidad de trabajo corresponde a una RPE 8-9 en la escala OMNI GSE, y la de descanso a un 3-4 (Seiler et al., 2004).

Los participantes realizaron tres entrenamientos distintos que se repitieron en el segundo microciclo, pero cambiando el ergómetro (el que se había hecho en bici de manos, se hacía en Skierg y viceversa).

Durante el tercer microciclo se incrementa la intensidad del primer y segundo entrenamiento (lunes y miércoles) un 3\%. En el tercer entrenamiento se aumenta la densidad, eliminando el minuto de descanso.

En el cuarto microciclo se repite el mismo entrenamiento que el tercero, pero cambiando de ergómetros.

A continuación, se detalla el entrenamiento en Tabla 3.

Para citar este artículo utilice la siguiente referencia: Rodríguez-Barcala, j.; Guerrero-Sánchez, 1.; Redón, X.. (2019). Influencia del entrenamiento interválico de alta intensidad en la capacidad aeróbica de personas con lesión medular. Sportis Sci J, 5 (2), 190-212. 
Artículo Original. Influencia del entrenamiento interválico de alta intensidad en la capacidad aeróbica de personas con lesión medular. Vol. $5, n^{\circ}$. 2; p. 190-212, mayo 2019. A Coruña. España ISSN 2386-8333

Tabla 3. Programa de entrenamiento

Primer día SKIERG

\begin{tabular}{|c|c|c|c|c|}
\hline$\frac{\sqrt{2}}{2}$ & 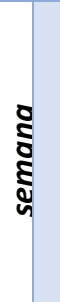 & $\begin{array}{l}3 \times 3 \times 1^{\prime} \\
\text { rec1' y } 2^{\prime}\end{array}$ & $\begin{array}{l}\mathbf{2 \times 8 ^ { \prime }} \\
\text { rec2' }\end{array}$ & $\begin{array}{l}\begin{array}{l}4 \times 60-60 \\
45-45 \\
30-30 \\
15-15\end{array} \\
\text { rec 1' }\end{array}$ \\
\hline & & Primer día - BICl & $\begin{array}{l}\text { Segundo día - } \\
\text { SKIERG }\end{array}$ & Tercer día - BICI \\
\hline 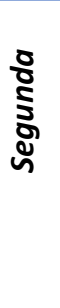 & 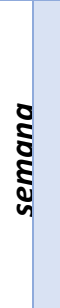 & $\begin{array}{c}3 \times 3 \times 1^{\prime} \\
\text { rec1' y } 2^{\prime}\end{array}$ & $\begin{array}{l}\mathbf{2 \times 8 ^ { \prime }} \\
\text { rec2' }\end{array}$ & $\begin{array}{l}\begin{array}{l}4 \times 60-60 \\
45-45 \\
30-30 \\
15-15\end{array} \\
\text { rec } 1^{\prime}\end{array}$ \\
\hline & & $\begin{array}{l}\text { Primer día - } \\
\text { SKIERG }\end{array}$ & $\begin{array}{l}\text { Segundo día - } \\
\text { BICI }\end{array}$ & Tercer día - SKIERG \\
\hline 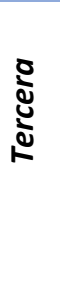 & 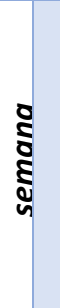 & $\begin{array}{c}3 \times 3 \times 1^{\prime} \\
\text { rec } 1^{\prime} 30^{\prime \prime} \text { y } 3^{\prime}\end{array}$ & $\begin{array}{l}\mathbf{3 \times 6} \mathbf{6}^{\prime} \\
\text { rec2' }\end{array}$ & $\begin{array}{l}\begin{array}{l}4 \times 60-60 \\
45-45 \\
30-30 \\
15-15\end{array} \\
\text { rec 1' }\end{array}$ \\
\hline & & Primer día - BICl & $\begin{array}{l}\text { Segundo día - } \\
\text { SKIERG }\end{array}$ & Tercer día - BICI \\
\hline$\frac{8}{0}$ & 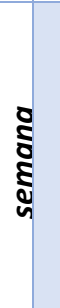 & $\begin{array}{c}3 \times 3 \times 1^{\prime} \\
\operatorname{rec} 1^{\prime} 30^{\prime \prime} \text { y } 3^{\prime}\end{array}$ & $\begin{array}{l}\mathbf{3 \times 6 ^ { \prime }} \\
\text { rec } 2^{\prime}\end{array}$ & $\begin{array}{l}\begin{array}{l}4 \times 60-60 \\
45-45 \\
30-30 \\
15-15\end{array} \\
\text { rec } 1^{\prime}\end{array}$ \\
\hline
\end{tabular}

Tercer día - SKIERG

Segundo día -

$\mathrm{BICI}$

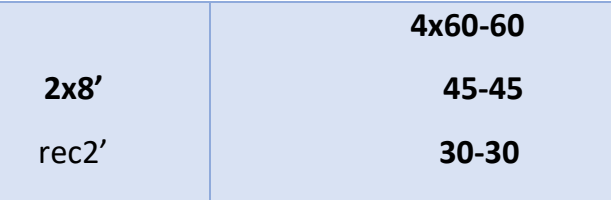

$$
\text { (1) }
$$


Artículo Original. Influencia del entrenamiento interválico de alta intensidad en la capacidad aeróbica de personas con lesión medular. Vol. $5, \mathrm{n}^{\circ}$. 2; p. 190-212, mayo 2019. A Coruña. España ISSN 2386-8333

El primer día de la semana, el entrenamiento se ejecutó al $75 \%$ del test de 1 minuto. El segundo día, al 95\% del test de 6': Y el tercer día en un 8-9 de una escala OMNI-GSE de percepción de esfuerzo.

En la tercera semana se dio la pauta de que incrementasen un $3 \%$ la intensidad en cada ergómetro.

Una vez terminadas las 12 sesiones, el primer día de la siguiente semana, se hizo un post-test, con todas las pruebas que se habían efectuado al principio de las 4 semanas.

Analisis de datos

El análisis de los datos se realizó mediante el paquete estadístico IBM SPSS Statistics v23. Se emplearon estadísticos descriptivos mediante medias y desviaciones típicas. Para el análisis de comparación de los datos obtenidos en el pretest y el post-test se utilizó la prueba t-Student para muestras relacionadas. Se asumió el 95\% como intervalo de confianza para la interpretación del análisis.

Variables antropométricas y aptitud cardiorrespiratoria antes y después de un programa interválico de alta intensidad

\section{Resultados}

Teniendo en cuenta el p valor, el peso, el perímetro de cintura y la potencia media relativa en un minuto, no son significativos. El resto de las variables son significativas con esta $\mathrm{n}$. Todos los datos significativos con un $\mathrm{p}$ valor $<0,05$ (sig.95\%).

Exponemos previamente una tabla descriptiva de los datos obtenidos antes y después del programa de entrenamiento. 
Artículo Original. Influencia del entrenamiento interválico de alta intensidad en la capacidad aeróbica de personas con lesión medular. Vol. $5, n^{\circ} .2$; p. 190-212, mayo 2019. A Coruña. España ISSN 2386-8333

Tabla 4. Valores descriptivos test inicial

Estadísticos descriptivos

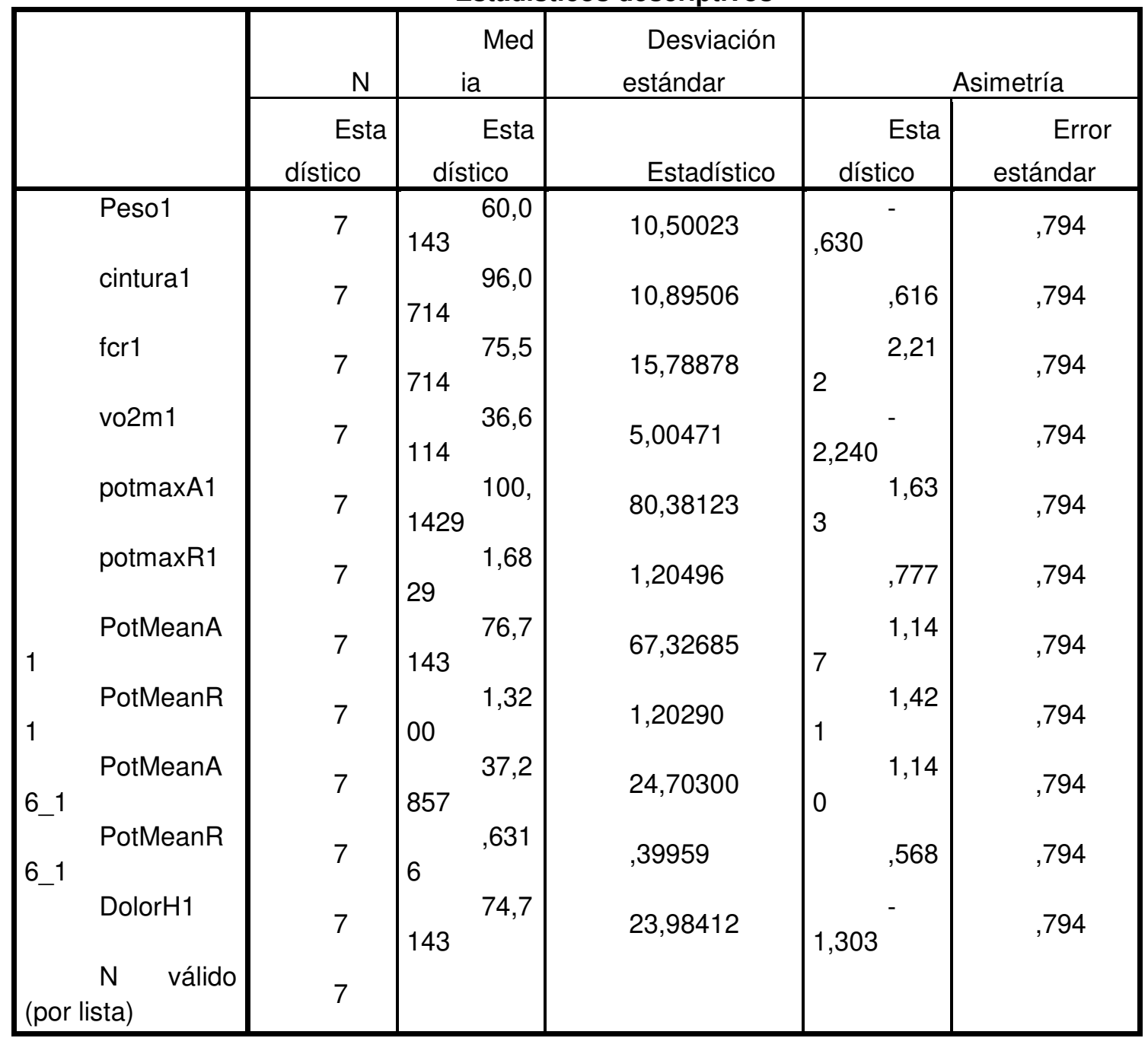


Artículo Original. Influencia del entrenamiento interválico de alta intensidad en la capacidad aeróbica de personas con lesión medular. Vol. 5, no. 2; p. 190-212, mayo 2019. A Coruña. España ISSN 2386-8333

Tabla 5. Valores descriptivos post-test

Estadísticos descriptivos

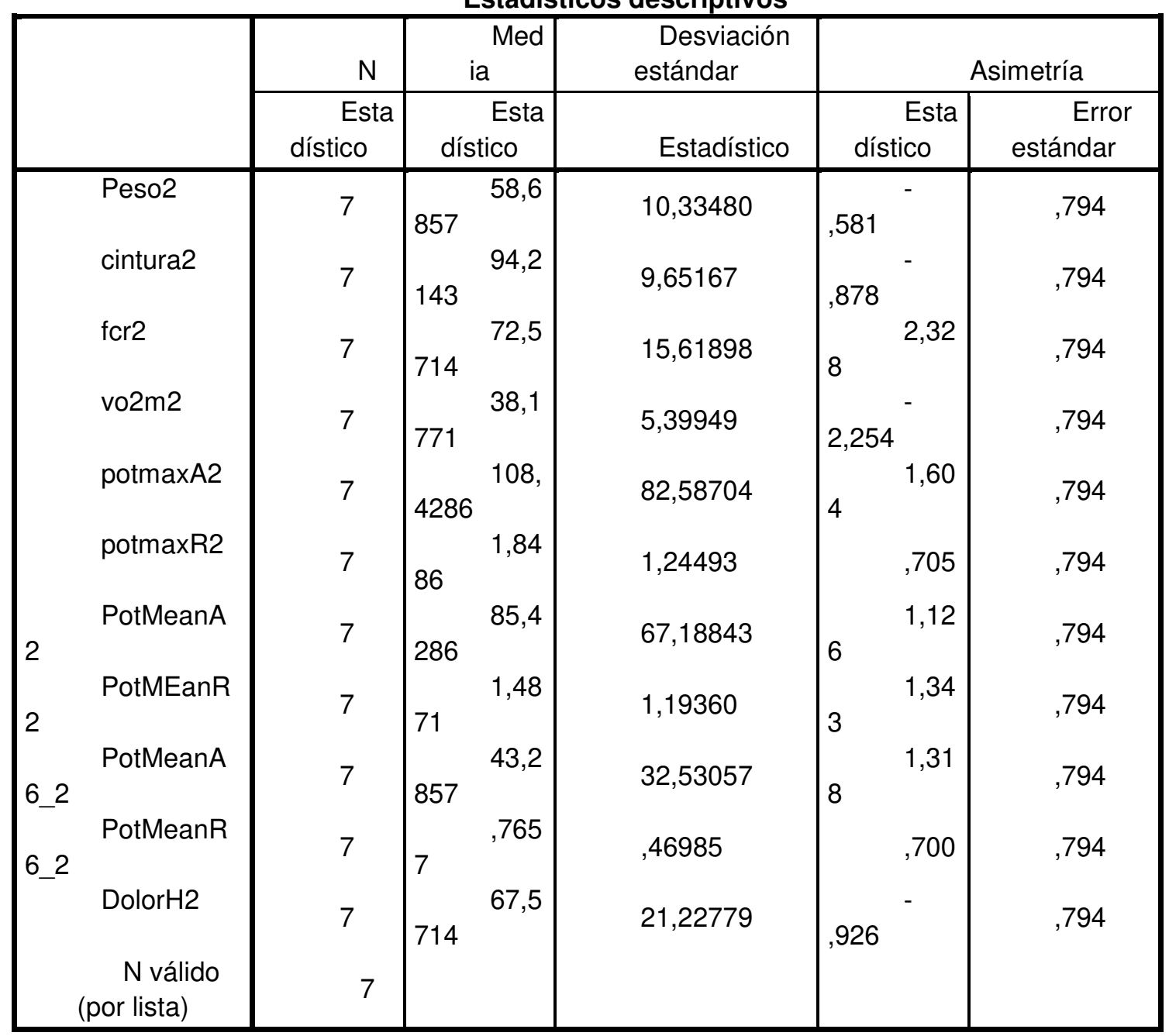


Artículo Original. Influencia del entrenamiento interválico de alta intensidad en la capacidad aeróbica de personas con lesión medular. Vol. 5, no . 2; p. 190-212, mayo 2019. A Coruña. España ISSN 2386-8333

Tabla 6. Variables antropométricas y de aptitud respiratoria antes y después del programa de entrenamiento

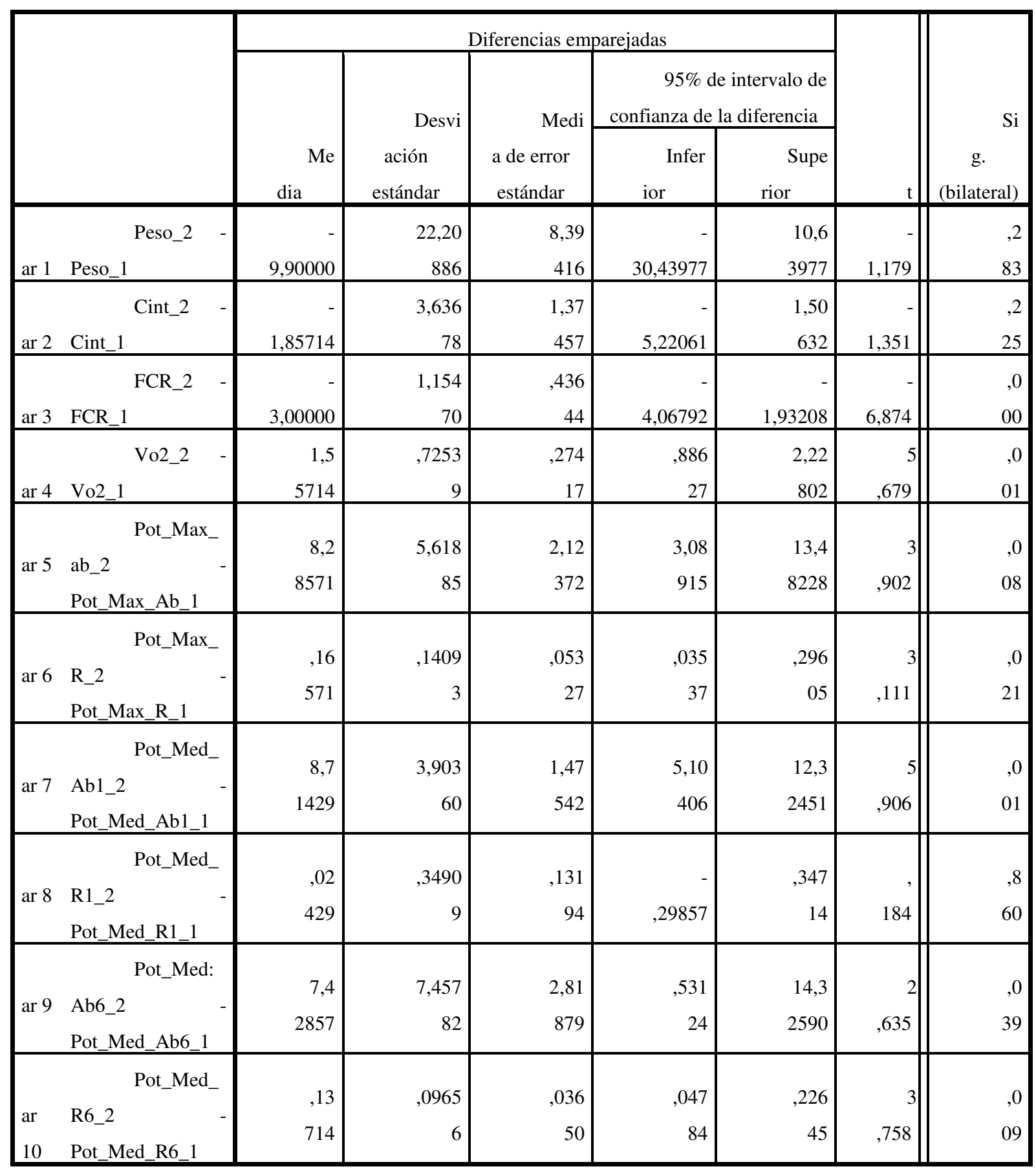


Artículo Original. Influencia del entrenamiento interválico de alta intensidad en la capacidad aeróbica de personas con lesión medular. Vol. $5, n^{\circ}$. 2; p. 190-212, mayo 2019. A Coruña. España ISSN 2386-8333

De todas las pruebas realizadas en el ergómetro, se produjo una mejora tanto en valores absolutos como relativos a su peso corporal.

La potencia máxima tanto absoluta como relativa mejora en todos los casos. La absoluta en 8 vatios, con una desviación estándar de $+/$ - 5 vatios, y la relativa, en 0,17 vatios por kg. de peso corporal (W/kg.) con una desviación estándar de +/- 0,14.

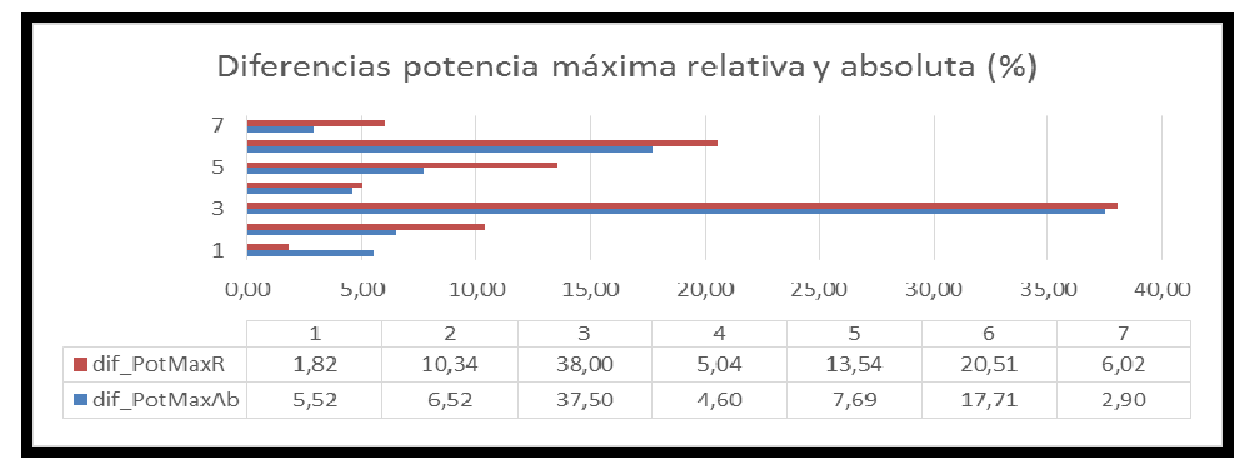

Figura 3. Diferencia en porcentaje de la potencia máxima relativa y absoluta 1_2

Por lo que respecta a la vía anaeróbica, la potencia media en un minuto, tanto absoluta como relativa mejora en todos los casos. La absoluta en 8 vatios, con una desviación estándar de $+/$ - 3 vatios, y la relativa en 0,02 vatios por $\mathrm{kg}$. de peso corporal (W/kg.) con una desviación estándar de +/- 0,34. Como ya se dijo, en periodos cortos de tiempo, es preferible tomar como referencia los valores absolutos, ya que los participantes con LM, pueden sufrir oscilaciones de peso, que alterarán el resultado (relativo a su peso corporal).

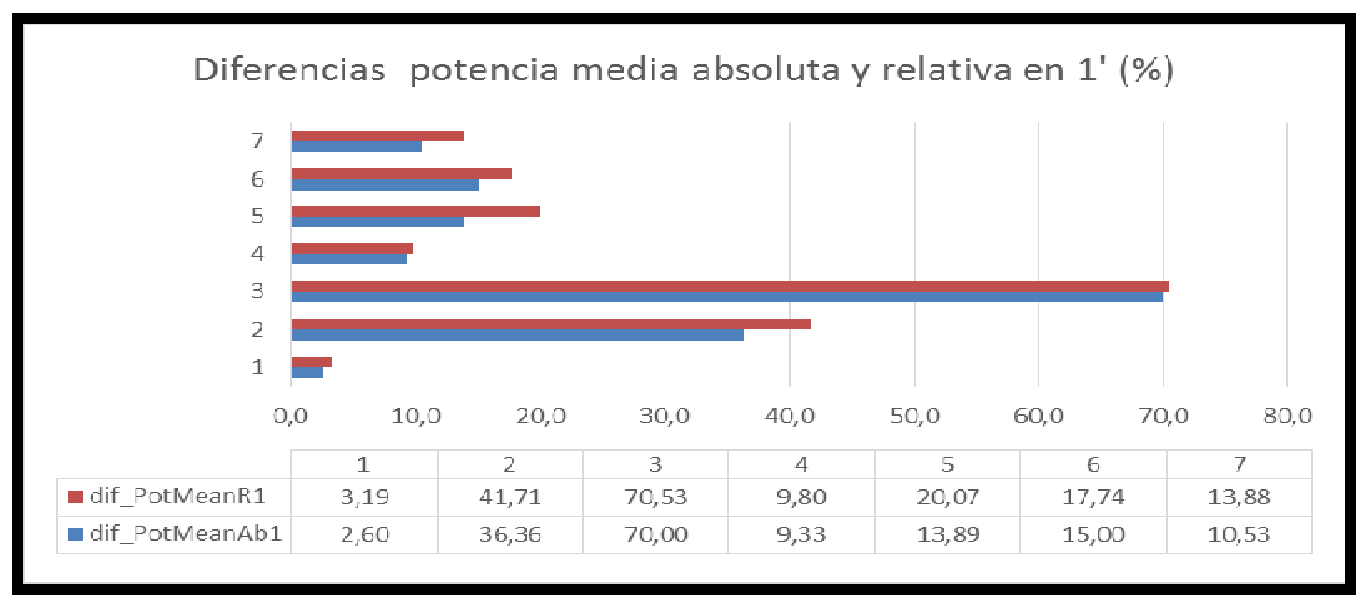

Figura 4. Diferencias en porcentaje de la potencia media absoluta y relativa en 1' $1 \_2$

Para citar este artículo utilice la siguiente referencia: Rodríguez-Barcala, j.; Guerrero-Sánchez, 1.; Redón, X.. (2019). Influencia del entrenamiento interválico de alta intensidad en la capacidad aeróbica de personas con lesión medular. Sportis Sci J, 5 (2), 190-212. DOI: https://doi.org/10.17979/sportis.2019.5.2.3919 
Artículo Original. Influencia del entrenamiento interválico de alta intensidad en la capacidad aeróbica de personas con lesión medular. Vol. $5, n^{\circ}$. 2; p. 190-212, mayo 2019. A Coruña. España ISSN 2386-8333

En cuanto a la vía aeróbica, la potencia media tanto absoluta como relativa mejora en todos los casos. La absoluta en 7 vatios, con una desviación estándar de +/ - 7 vatios, y la relativa, en 0,13 vatios por $\mathrm{kg}$. de peso corporal (W/kg.) con una desviación estándar de +/0,09 .

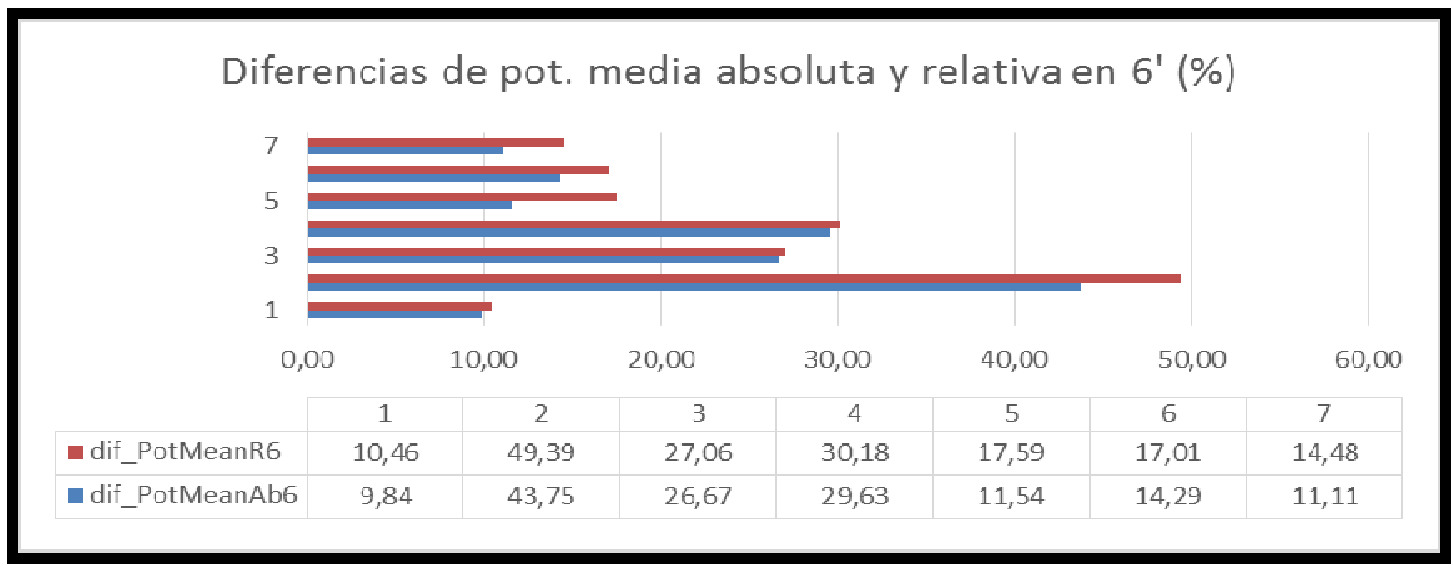

Figura 5. Diferencias en porcentaje de la potencia media absoluta y relativa en 6' 1_2

En la estimación que hacemos del VO2max, se produce una mejora de $1.5 \mathrm{ml} / \mathrm{kg} / \mathrm{min}$, con una desviación estándar de $+/-0.7 \mathrm{ml} / \mathrm{kg} / \mathrm{min}$. Hemos visto que uno de los principales problemas en los participantes con LM es que no llegan al nivel mínimo de VO2max para realizar las actividades de la vida diaria (AVD). En este caso, con 12 sesiones de entrenamiento interválico de alta intensidad, los participantes mejoraron entre un 2.8 y un $7.2 \%$ su VO2max estimado.

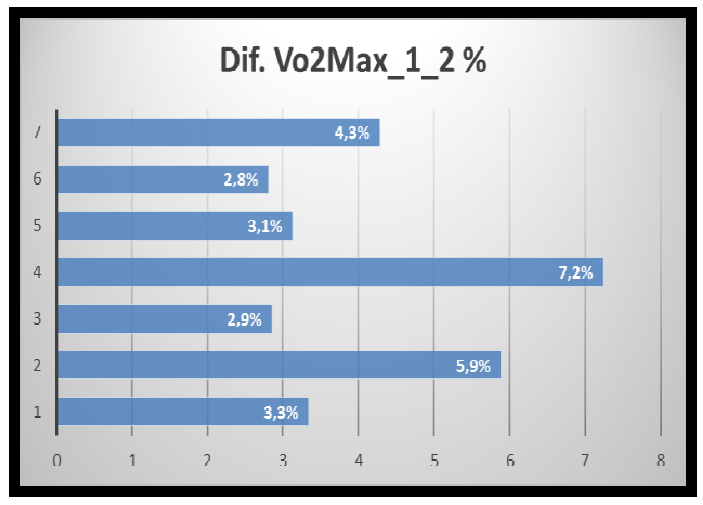

Figura 6. Diferencias en porcentaje de $\mathrm{VO}_{2}$ máx

La Frecuencia cardiaca de reposo, que mejoró en todos los casos, lo hizo en -3 ppm, con una desviación estándar de 1,15 ppm.

Para citar este artículo utilice la siguiente referencia: Rodríguez-Barcala, j.; Guerrero-Sánchez, 1.; Redón, X.. (2019). Influencia del entrenamiento interválico de alta intensidad en la capacidad aeróbica de personas con lesión medular. Sportis Sci J, 5 (2), 190-212. 
Artículo Original. Influencia del entrenamiento interválico de alta intensidad en la capacidad aeróbica de personas con lesión medular. Vol. $5, n^{\circ}$. 2; p. 190-212, mayo 2019. A Coruña. España ISSN 2386-8333

Como ya se citó antes, tanto el peso, como perímetro de cintura, no son significativos.

Analizando los resultados obtenidos en la versión española del cuestionario DASH (Hervás, Navarro, Peiró, Rodrigo, López y Martínez, 2006), consistente en 30 preguntas con una puntuación entre 1 y 5 cada una (donde 1 es poco dolor y capacidad total y 5 es una incapacidad total).

Podemos decir que tras el programa de entrenamiento se ha reducido el dolor de hombro entre un 6 y 17\% según los resultados obtenidos en el cuestionario. Todo ello con una significación de un 95\% para esta n. (Ver anexo 1. Cuestionario DASH sobre las discapacidades del hombro, codo y mano).
\end{abstract}

\title{
Discusión y conclusiones
}

Este estudio estuvo motivado por la necesidad de comprobar si el entrenamiento interválico de alta intensidad produce una mejora en la capacidad aeróbica y en el dolor de hombro, independientemente del tiempo que ha pasado desde que se produjo la lesión y la altura de la misma. Hemos elegido un trabajo interválico en lugar de uno continuo para reducir el volumen de entrenamiento y disminuir la carga articular (Guillen and Gibala, 2014).

Tal y como se referencia en el estudio de Buchholz et al. (2009), la actividad física representa un papel importante en la prevención primaria y secundaria de muchos problemas asociados a la LM (enfermedades cardiovasculares, diabetes, hipertensión, dislipemias...). Más concretamente, Hetz, Hetz, Latimer, Buchholz, Martin y SHAPE-SCI Research Group (2009) objetivaron que las personas con LM que realizan mayor cantidad de AF son menos proclives a desarrollar resistencia a la insulina, tienen menos riesgo de hipertensión y una menor circunferencia abdominal.

Uno de los síntomas más comunes en las personas con LM es el dolor de la articulación del hombro (Goldstein, Young y Escobedo, 1997) El motivo de aparición de este síntoma más aceptado entre los expertos en la falta de fuerza del hombro (Penthland y Twomey,1999; Burnham, Nelson, Steadward, Reid 2012), unido a su sobreuso en las actividades diarias.

Para citar este artículo utilice la siguiente referencia: Rodríguez-Barcala, j.; Guerrero-Sánchez, 1.; Redón, X.. (2019). Influencia del entrenamiento interválico de alta intensidad en la capacidad aeróbica de personas con lesión medular. Sportis Sci J, 5 (2), 190-212.

DOI: https://doi.org/10.17979/sportis.2019.5.2.3919

http://revistas.udc.es/ 
Artículo Original. Influencia del entrenamiento interválico de alta intensidad en la capacidad aeróbica de personas con lesión medular. Vol. $5, n^{\circ}$. 2; p. 190-212, mayo 2019. A Coruña. España ISSN 2386-8333

Los trabajos desarrollados hasta el momento han observado una disminución del dolor y un aumento de la funcionalidad tras la realización de ejercicios de fuerza (Jacobs y Nash, 2004; Serra-Añó et al., 2012; Nash, van de Ven, van Elk y Johnson, 2007). Por ello, la adherencia a un programa específico donde se pautan ejercicios compensatorios de hombro mejora el pronóstico de la articulación, ya que aumenta la fuerza y la movilidad, reduciendo así el dolor.
\end{abstract}

Las personas con LM presentan una "hipocinesia circulatoria" que genera una acumulación de sangre venosa en los miembros inferiores y frecuentemente en la parte baja del tronco, debido al escaso movimiento de estos miembros, así como al control limitado del sistema nervioso (SN) especialmente el SN simpático, por debajo del nivel de la lesión (Jehl et al., 1991; Hopman et al., 2002; extraído de Brizuela et al. 2016). Por esta razón, proponemos utilizar los valores absolutos y no los relativos a su peso corporal.

Debido a esta alteración, existe una disminución del gasto cardíaco y del volumen sistólico, viéndose aumentada esta alteración con la altura de la lesión, debido muy probablemente a que el deficitario retorno venoso no favorece el llenado ventricular. Sin embargo, a intensidades submáximas, esto se compensa con el aumento de la FC, siempre que se mantenga intacta la inervación simpática del corazón (Hopman et al., 1993).

En pruebas de resistencia se verá disminuido el rendimiento, cuando la intensidad esté cercana al máximo, debido a que la capacidad de transportar de oxígeno a los tejidos está limitada.

Si la lesión está por debajo de T6-T7, la inervación cardiaca y la vascular del área renal y esplácnica se preservan al completo y la función cardíaca no está alterada, con una FC proporcionada a la intensidad del ejercicio.

En deportistas con LM más alta que T6, su FC se verá limitada y resultará inferior a lo previsible. Ésta puede mostrarse tardía en cuanto a su tiempo de subida, y limitada en su magnitud, ya que, debido a la escasa actividad de la musculatura respiratoria, no permite aprovechar su efecto de "bomba" que contribuye al retorno venoso, al incremento de la precarga cardíaca y al incremento del VS (Bhambhani, 2002).

Por tanto, King (citado en Brizuela, 2016) nos explica que deberán dedicar un tiempo mayor al calentamiento, para asegurar la puesta en marcha de su sistema cardiovascular y

Para citar este artículo utilice la siguiente referencia: Rodríguez-Barcala, j.; Guerrero-Sánchez, 1.; Redón, X.. (2019). Influencia del entrenamiento interválico de alta intensidad en la capacidad aeróbica de personas con lesión medular. Sportis Sci J, 5 (2), 190-212. 
Artículo Original. Influencia del entrenamiento interválico de alta intensidad en la capacidad aeróbica de personas con lesión medular. Vol. $5, n^{\circ}$. 2; p. 190-212, mayo 2019. A Coruña. España ISSN 2386-8333

evitar una deuda de oxígeno inicial, además de contribuir a conseguir una la tensión arterial adecuada, normalmente baja en personas con tetraplejia, evitando desencadenar alteraciones cardíacas funcionales y estructurales

Estos deportistas (lesión más alta de T6) tienen una serie de alteraciones importantes del sistema nervioso autónomo que provocarán cambios en la coagulación, el metabolismo de la insulina, degenerativos articulares y en la densidad mineral ósea (Nash et al., 1994). Existe una reducción de la densidad mineral ósea predominante durante el primer año tras la lesión (Uebekheart et al., 1995).

Debido a inestabilidad en el tronco que sufren las personas con LM, que se ve aumentada con la altura de la lesión, estos test de potencia y/o fuerza máxima requieren de un aprendizaje. En nuestro caso no fue necesario, ya que todos los participantes tenían más de dos años de experiencia previa, pero es un factor a tener en cuenta en futuras investigaciones

Por todo lo expuesto, se recomienda que las personas con LM participen en actividades deportivas, dado que existe una mejoría en la potencia aeróbica y anaeróbica, en la funcionalidad y calidad de vida; así como en su interacción social, independencia, movilidad y disminución del estrés (Slater y Meade, 2004).

Además, aconsejamos el uso de los vatios como medida de referencia más precisa para cuantificar la intensidad del entrenamiento en participantes con lesión medular, en lugar de usar la FC, ya que ésta tiene una respuesta demasiado variable en función del tipo y altura de la lesión y del propio individuo.

El entrenamiento interválico de alta intensidad resulta una buena estrategia para mejorar la fuerza absoluta de participantes con lesión medular, su resistencia cardiovascular a nivel global, resistencia muscular. Además, se observa una tendencia a la disminución de dolor en el hombro, pudiendo desarrollar más actividades en su día a día, mejorando su calidad de vida consecuentemente.

\section{Aplicación práctica}

Con este trabajo mostramos que el entrenamiento interválico de alta intensidad es una herramienta totalmente válida para personas con lesión medular a la hora de mejorar los parámetros relacionados con la resistencia cardiovascular, la fuerza y las patologías de hombro.

Para citar este artículo utilice la siguiente referencia: Rodríguez-Barcala, j.; Guerrero-Sánchez, 1.; Redón, X.. (2019). Influencia del entrenamiento interválico de alta intensidad en la capacidad aeróbica de personas con lesión medular. Sportis Sci J, 5 (2), 190-212. DOI: https://doi.org/10.17979/sportis.2019.5.2.3919

http://revistas.udc.es/ 


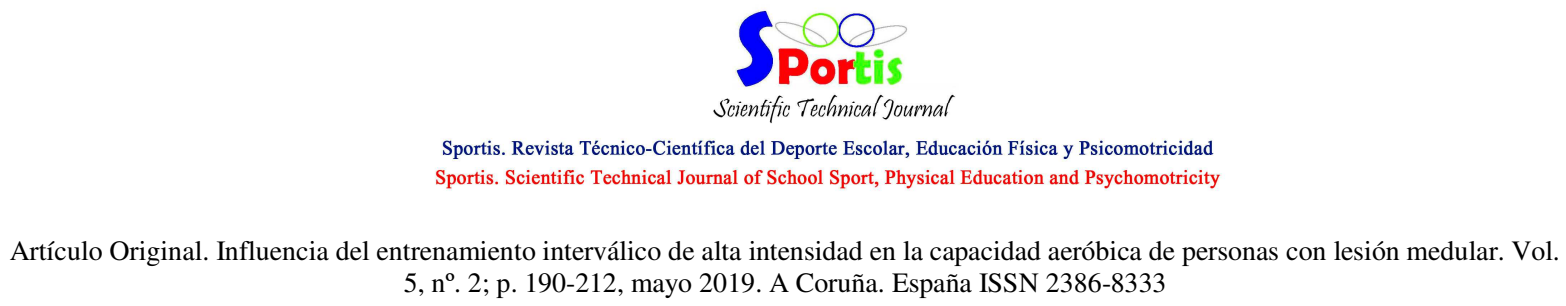

Los resultados obtenidos indican que este tipo de entrenamiento es una forma válida y sencilla de aplicar para cualquier entrenador. Además, no se necesita maquinaria especial, con lo que se puede llevar a cabo en cualquier instalación deportiva de fitness.

El hecho de haber obtenido resultados positivos en personas con LM físicamente activas invita a pensar que los resultados en personas sedentarias pueden ser aún mayores.

Vista la total adherencia al entrenamiento interválico que hubo en la muestra del presente estudio, nos hace pensar que un tipo de entrenamiento de estas características (duración de entre 10-20 minutos y con alternancias de ritmos e intensidades) podría resultar más atractivo que uno continuo de larga duración, no solo en personas activas sino también en personas sedentarias.

Es difícil demostrar a nivel estadístico el resultado, debido a que la variedad de lesiones hace que los perfiles de los participantes sean muy distintos, ya que la n es muy baja y, a pesar de que todos los englobemos en lesión medular para este trabajo.

\section{Referencias bibliográficas}

Bhambhani, Y. N. (2002). Physiology of wheelchair Racing in athletes with spinal cord injury. Sports Medicine, 32(1): 23-51.

Brizuela, G.; Sinz, S.; Aranda, R., y Martínez, I. (2010). Efecto del pedaleo de brazos sobre el sistema cardiorrespiratorio de las personas con tetraplejia. RICYDE. Revista internacional de ciencias del deporte, 21(6), 297-310. http://dx.doi.org/10.5232/ricyde2010.02104.

Brizuela, G. et al. (2016). Lesión medular y ejercicio físico: revisión desde una perspectiva deportiva. Revista Española de Discapacidad, 4 (2), 163-185.

Buccholz A.C. et al. (2009). Greater daily leisure time physical activity is associated with lower chronic disease risk in adults with spinal cord injury. Appl. Physiol.Nutr. Metab. $34,640-647$.

Para citar este artículo utilice la siguiente referencia: Rodríguez-Barcala, j.; Guerrero-Sánchez, 1.; Redón, X.. (2019). Influencia del entrenamiento interválico de alta intensidad en la capacidad aeróbica de personas con lesión medular. Sportis Sci J, 5 (2), 190-212. DOI: https://doi.org/10.17979/sportis.2019.5.2.3919

http://revistas.udc.es/ 
Artículo Original. Influencia del entrenamiento interválico de alta intensidad en la capacidad aeróbica de personas con lesión medular. Vol. $5, \mathrm{n}^{\circ}$. 2; p. 190-212, mayo 2019. A Coruña. España ISSN 2386-8333

Burnham, R.S., May, L., Nelson, E., Steadward, R. \& Reid, D. C. (2012) Shoulder functionality in paraplegics. Spinal Cord 50, 827-831.

Clarkson, H. (2013). Proceso evaluativo musculoesquelético. Paidotribo. (120-177).

Da Silva-Grigoletto, M.E., Viana-Montaner, B.H., Heredia, J.R., Mata, F., Peña, G., Brito, C.J., Vaamonde, D., García-Manso, J.M. (2013). Validación de la escala de valoración subjetiva del esfuerzo OMNI-GSE para el control de la intensidad global en sesiones de objetivos múltiples en personas mayores. Kronos XII (1), 32-40.

Giner, M. (2006): Guía de autocuidados para lesionados medulares, Valencia: Generalitat Valenciana, Consellería de Sanitat. 54-56.

Finley, M. A. y Rodgers, M. M. (2004): "Prevalence and identification of shoulder pathology in athletic and nonathletic wheelchair users with shoulder pain: a pilot study”. Journal of Rehabilitation Research and Development, 41 (3B): 395-402.

Fullerton, H. D. et al. (2003): "Shoulder pain: a comparison of wheelchair athletes and nonathletic wheelchair users". Medicine and science in sports and exercise, 35 (12): 1958-1961.

Hastings, J., Goldstein, B. \& Escobedo, E. (1997) Rotator cuff repairs in individuals with paraplegia. Am. J. Phys. Med. Rehabil.

Hazel M Clarkson. (2003). Proceso evaluativo músculo esquelético, pág.125.

Hervás, M. T., Navarro Collado, M. J., Peiró, S., Rodrigo Pérez, J.L., López Matéu, P., Martínez Tello, I (2006). Versión española del cuestionario DASH. Adaptación transcultural, fiabilidad, validez y sensibilidad a los cambios. Medicina Clínica, 127(12):441-7.

Para citar este artículo utilice la siguiente referencia: Rodríguez-Barcala, j.; Guerrero-Sánchez, 1.; Redón, X.. (2019). Influencia del entrenamiento interválico de alta intensidad en la capacidad aeróbica de personas con lesión medular. Sportis Sci J, 5 (2), 190-212. 


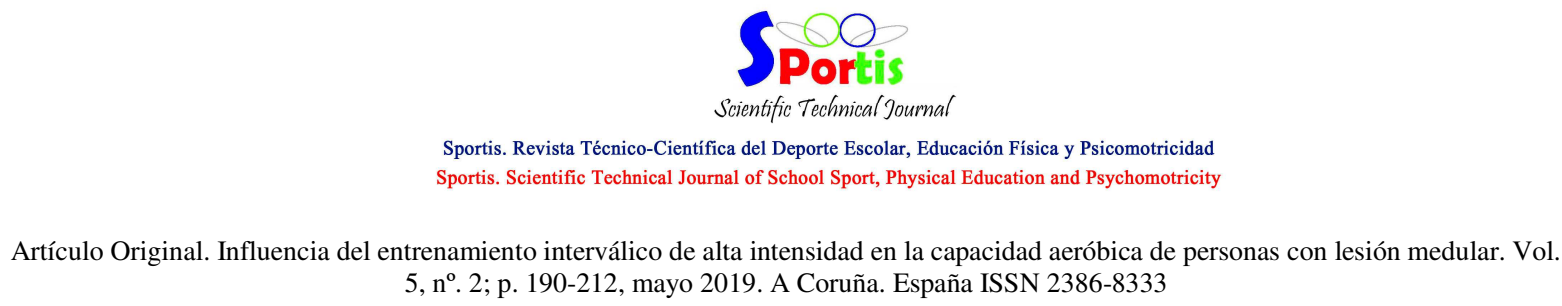
5, no. 2; p. 190-212, mayo 2019. A Coruña. España ISSN 2386-8333

Hetz, S.P., Latimer, A.E., Buchholz, A.C., Martin Ginis, K.A. \& SHAPE-SCI Research Group (2009). Increased participation in activities of daily living is associated with lower cholesterol levels in people with spinal cord injury. Arc. Phys. Med. Rehabil. 90, 1755-1759. At <http://soundideas.pugetsound.edu/faculty_pubs/1202>.

Hicks, A.L.; Martin, K.A.; Ditor, D.S.; Latimer, A.E.; Craven, C.; Bugaresti, J., \& McCartney, N. (2003). Long-term exercise training in persons with spinal cord injury: Effects on strength, arm ergometry performance and psychological wellbeing. Spinal Cord, 41(1), 34-43. http://dx.doi.org/10.1038/sj.sc.3101389.

Hicks, A.L.; Martin, K.A.; Pelletier, C.A.; Ditor, D.S.; Foulon, B., \& Wolfe, D. L. (2010). The effects of exercise training on physical capacity, strength, body composition and functional performance among adults with spinal cord injury: A systematic review. Physical Medicine and Rehabilitation, 91(5), 729-733.

Hjeltnes, N. and Jansen, T. (1990). Physical endurance capacity, functional status and medical complications in spinal cord injured subjects with long-standing lesions. Paraplegia. $28(7), 428-432$.

Jacobs, D. P.L. \& Nash M.S. (2004) Exercise recommendations for individuals with spinal cord injury. Sports Medicine 34, 727-751.

Kohl, H.W., 3rd. (2001). Physical activity and cardiovascular disease: evidence for a dose response. Med Sci Sports Exerc. 33(6 Suppl), S472-483.

Marins, J.C.B.; Delgado, M.; Benito, P.J; (2013): Precisión de las ecuaciones para estimar la frecuencia cardíaca máxima en cicloergómetro. Archivos de Medicina del deporte, 1420.

Para citar este artículo utilice la siguiente referencia: Rodríguez-Barcala, j.; Guerrero-Sánchez, 1.; Redón, X.. (2019). Influencia del entrenamiento interválico de alta intensidad en la capacidad aeróbica de personas con lesión medular. Sportis Sci J, 5 (2), 190-212. DOI: https://doi.org/10.17979/sportis.2019.5.2.3919

http://revistas.udc.es/ 
Artículo Original. Influencia del entrenamiento interválico de alta intensidad en la capacidad aeróbica de personas con lesión medular. Vol. $5, n^{\circ}$. 2; p. 190-212, mayo 2019. A Coruña. España ISSN 2386-8333

McDonald M. J. (2016). Predicting peak oxygen uptake from submaximal exercise after spinal cord injury. Applied Physiology, Nutrition, and Metabolism. Recuperado de https://mc06.manuscriptcentral.com/apnm-pubs.

Myers, J., Lee, M., and Kiratli, J. (2007). Cardiovascular disease in spinal cord injury: an overview of prevalence, risk, evaluation, and management. Am J Phys Med Rehabil. $86(2), 142-152$.

Nash, M.S., van de Ven, I, van Elk, N. \& Johnson. B. M (2007) Effects of circuit resistance training on fitness attributes and upper- extremity pain in middle-aged men with paraplegia. Arch. Phys. Med. Rehabil. 88, 70-75.

Pentland, W.E. \&Twomey, L. T. (1999) The weight-bearing upper extremity in women with long term paraplegia. Spinal cord 37. 421-429.

Serra-Añó, P. et al. (2012). Effects of resistance training on strength, pain and shoulder functionality in paraplegic. Spinal Cord 50, 827-831.

Slater, D. \& Meade, M. A. (2004). Participation in recreation and sports for persons with spinal cord injury. Review and recommendations. Neurorehabilitation 19, 121-129.

Uebelhart, D., Demiaus-Domenech, B. Roth, M. \& Chantraine, A. (1995). Bone metabolism in spinal cord injured individuals and in others who have prolonged immobilization. A review. Spinal Cord 33, 669-673.

Uth N, Sørensen H, Overgaard K, Pedersen PK (2004). Estimation of VO2max from the ratio between HRmax and Hrrest- the heart rate ratio method. Eur J Appl Physiol. 004;91(1):111-115. 
Artículo Original. Influencia del entrenamiento interválico de alta intensidad en la capacidad aeróbica de personas con lesión medular. Vol. $5, n^{\circ}$. 2; p. 190-212, mayo 2019. A Coruña. España ISSN 2386-8333

Valent, L.J.M.; Dallmeijer, A.J.; Houdijk, H.; Slootman, J.H.; Janssen, T.W., \& van der Woude, L.H.V. (2009). Effects of hand cycle training on physical capacity in individuals with tetraplegia: A clinical trial. Physical Activity and Therapy, 89, 10511060. http://dx.doi.org/10.2522/ptj.20080340.

Van Koppenhagen, C.F., Post, M., de Groot, S., van Leeuwen, C., van Asbeck, F., StolwijkSwuste, J., van der Woude, L., and Lindeman, E. (2014). Longitudinal relationship between wheelchair exercise capacity and life satisfaction in patients with spinal cord injury: A cohort study in the Netherlands. The journal of spinal cord medicine. 37(3), 328-337.

\section{Agradecimientos}

A todos los deportistas que han participado en este estudio.

A todos los técnicos de los Centros Puente de la Fundació Isidre Esteve, que han llevado a cabo todos los entrenamientos, tanto en Vigo como en Sant Cugat.

A todo el grupo de trabajo Fundació Isidre Esteve Research 4. (FIER 4) 